\title{
EXPLICIT SOLUTIONS OF GENERALIZED NONLINEAR BOUSSINESQ EQUATIONS
}

\section{DOĞAN KAYA}

Received 13 September 2000

By considering the Adomian decomposition scheme, we solve a generalized Boussinesq equation. The method does not need linearization or weak nonlinearly assumptions. By using this scheme, the solutions are calculated in the form of a convergent power series with easily computable components. The decomposition series analytic solution of the problem is quickly obtained by observing the existence of the self-canceling "noise" terms where sum of components vanishes in the limit.

\section{Introduction}

A generalized Boussinesq equation

$$
u_{t t}-[D(u)]_{x x}-u_{x x x x}=f(x, t),
$$

where $\mathrm{D}(\mathfrak{u})$ is an arbitrary sufficiently differentiable function, with the condition that $[D(u)]_{x x} \neq 0$ to ensure nonlinearity and $f(x, t)$ is a given function. The initial conditions are given in the form of $u(x, 0)=g_{1}(x)$, $u_{\mathrm{t}}(x, 0)=g_{2}(x)$.

Equation (1.1) has been proposed as a model for propagation of pulses along a transmission line made of a large number of LC-circuits and as a model to describe vibrations of a single one-dimensional dense lattice [15]. However, in each of these studies, Rosenau remarks that (1.1) is ill-posed, and additional assumptions must be made on the nonlinearity of $\mathrm{D}(\mathfrak{u})$ [13].

A classification of (1.1) is undertaken by applying both the classical method of Lie and the nonclassical method of Bluman and Cole [5]. This class of nonlinear Boussinesq equation has previously been studied in some of the references $[9,10,13]$. Clarkson and Kruskal [10] introduced some 
similarity reduction of the Boussinesq equation. These symmetry reductions are obtained using the direct method. By using this method, the equation is reduced to the first, second, and fourth Painleve equations, which involves no group theoretic techniques. Another significant work to find exact solutions of the Boussinesq equation is studied by Clarkson [9]. He stated that the solutions of this equation are obtained in two different ways: one of these, by using the classical and nonclassical reductions of the equations to find the corresponding ordinary differential equations, which are solvable in terms of the first, second, and fourth Painleve equations. The exact solutions are generated from these ordinary differential equations. The second way, he used new space independent similarity reductions of the Boussinesq equation. He also generated both the second and fourth Painleve equations for finding the exact solutions of the equation by using this similarity reductions. In [13], Clarkson and Priestly found conditions on $\mathrm{D}(\mathrm{u})$ such that it allows symmetries, in particular those beyond the translation symmetries of the independent variables. They used the classical Lie method, and the nonclassical method, to find these symmetries. Once the symmetries of (1.1) have been found, they find the associated reductions and test the ordinary differential equations and then solve the equation. However, the application of the methods are not entirely straightforward [13].

In this paper, the nonlinear equation (1.1) with three cases of the nonlinear term of $\mathrm{D}(\mathrm{u})$ which are given in [13], homogeneous or inhomogeneous, will be handled more easily, quickly, and elegantly by implementing the Adomian's decomposition method $[1,2,3]$ rather than the traditional methods for the explicit solutions. In this paper, we do not use any reductions or transformation to reduce problem (1.1) to an ordinary differential equation, a system of simpler partial differential equations or any linearization, perturbation scheme. The original nonlinear equation is directly solvable preserving the actual physics and involving much less calculation [3]. The decomposition scheme is illustrated by studying problem (1.1) to compute approximate solution for this problem. Furthermore, we also illustrate selfcanceling phenomena for problem (1.1) using the decomposition method.

\section{The method of solution}

In this section, we outline the method to obtain explicit solution of problem (1.1) using the decomposition method. We consider problem (1.1) in an operator form

$$
L_{t}(u)-N(u)-L_{x}(u)=f(x, t),
$$

where the notation $N(u)=[D(u)]_{x x}$ symbolizes the nonlinear term, the notations $E_{t}=\partial^{2} / \partial t^{2}$ and $E_{x}=\partial^{4} / \partial x^{4}$ symbolize the linear differential operators. Assuming that the inverse of the operator $E_{t}^{-1}$ exists and it can 
conveniently be taken as the two-fold definite integral with respect to $t$ from 0 to $t$, that is, $E_{t}^{-1}=\int_{0}^{t} \int_{0}^{t}(\cdot) d t d t$. Thus, applying the inverse operator $E_{t}^{-1}$ to (2.1) yields

$$
\mathrm{L}_{\mathrm{t}}^{-1} \mathrm{~L}_{\mathrm{t}}(\mathrm{u})=\mathrm{L}_{\mathrm{t}}^{-1}(\mathrm{f}(\mathrm{x}, \mathrm{t}))+\mathrm{L}_{\mathrm{t}}^{-1}(\mathrm{~N}(\mathrm{u}))+\mathrm{L}_{\mathrm{t}}^{-1} \mathrm{~L}_{\mathrm{x}}(\mathrm{u}) .
$$

Therefore, it follows that

$$
u(x, t)=u(x, 0)+t u_{t}(x, 0)+L_{t}^{-1}(f(x, t))+L_{t}^{-1}(N(u))+L_{t}^{-1} L_{x}(u) .
$$

We obtain the zeroth component by

$$
u_{0}=g_{1}(x)+\operatorname{tg}_{2}(x)+L_{t}^{-1}(f(x, t))
$$

which is defined by all terms that arise from the initial conditions, and from integrating the source term and decompose the unknown function $u(x, t), a$ sum of components defined by the decomposition series

$$
u(x, t)=\sum_{n=0}^{\infty} u_{n}(x, t)
$$

and the nonlinear term $N(u)=[D(u)]_{x x}$ is expressed in the form

$$
\mathrm{N}(\mathfrak{u})=[\mathrm{D}(\mathfrak{u})]_{\mathrm{xx}}=\sum_{\mathfrak{n}=0}^{\infty} A_{\mathfrak{n}},
$$

where $A_{n}$ are called the Adomian polynomials as calculated by $[2,18]$ according to specific algorithms. One can use the general form of the formula for $A_{n}$ Adomian polynomials as follow:

$$
\begin{aligned}
& A_{0}=\left[D\left(u_{0}\right)\right]_{x x}, \\
& A_{1}=\left[u_{1}\left(\frac{\partial}{\partial u_{0}}\right) D\left(u_{0}\right)\right]_{x x}, \\
& A_{2}=\left[u_{2}\left(\frac{\partial}{\partial u_{0}}\right) D\left(u_{0}\right)+\left(\frac{u_{1}^{2}}{2 !}\right)\left(\frac{\partial^{2}}{\partial u_{0}^{2}}\right) D\left(u_{0}\right)\right]_{x x}, \\
& A_{3}=\left[u_{3}\left(\frac{\partial}{\partial u_{0}}\right) D\left(u_{0}\right)+u_{1} u_{2}\left(\frac{\partial^{2}}{\partial u_{0}^{2}}\right) D\left(u_{0}\right)+\left(\frac{u_{1}^{3}}{3 !}\right)\left(\frac{\partial^{3}}{\partial u_{0}^{3}}\right) D\left(u_{0}\right)\right]_{x x},
\end{aligned}
$$

and so on for other polynomials. A variation of the generalized Adomian polynomials can be found in $[2,18]$. 
With the zeroth component defined as above, the remaining components $u_{n}(x, t), n \geq 1$, can be completely determined such that each term is computed by using the previous term. Since $\mathfrak{u}_{0}$ is known,

$$
\begin{aligned}
u_{1} & =L_{t}^{-1}\left(A_{0}\right)+L_{t}^{-1} L_{x}\left(u_{0}\right), \\
u_{2} & =L_{t}^{-1}\left(A_{1}\right)-L_{t}^{-1} L_{x}\left(u_{1}\right), \\
& \vdots \\
u_{n} & =L_{t}^{-1}\left(A_{n-1}\right)-L_{t}^{-1} L_{x}\left(u_{n-1}\right),
\end{aligned}
$$

for $n \geq 0$. It is useful to note that the recursive relationship is constructed on the basis that the zeroth component $u_{0}(x, t)$ is defined by all terms that arise from the initial condition and from integrating the source term. The remaining components $u_{n}(x, t), n \geq 0$, can be completely determined such that each term is computed by using the previous term. As a result, the components $\mathfrak{u}_{0}, \mathfrak{u}_{1}, \mathfrak{u}_{2}, \ldots$ are identified and the series solutions are thus entirely determined. However, in many cases the exact solution in a closed form may be obtained.

Several authors have investigated the convergence of the Adomian decomposition method. The theoretical treatment of convergence of the decomposition method has been considered by Cherruault [7] and Rèpaci [14]. In [7], Cherruault proposed a new definition of the technique and then he insisted that it will become possible to prove the convergence of the decomposition method. Rèpaci [14] showed a convergence of this method based upon a suitable connection with fixed point techniques. This is essentially the same conclusion derived by Cherruault [7]. These results have been improved by Cherruault and Adomian [8], who proposed a new convergence proof of Adomian's technique based on properties of convergent series. They obtained some results about the speed of convergence of this method enabling us to solve nonlinear functional equations. To give a clear overview of the methodology, we have selected a variety of illustrative examples.

\section{Test problems}

Problem 1. We first consider, setting $D(u)=(1 / 2) u^{2}$ in (1.1) yields the Boussinesq equation

$$
\mathfrak{u}_{\mathrm{tt}}=\left[\frac{1}{2} \mathfrak{u}^{2}\right]_{x x}+\mathfrak{u}_{x x x x}+f(x, t)
$$

which is a solution equation solvable by inverse scattering [11, 19], originally used by Boussinesq [6] to describe the propagation of long waves in shallow water. We would like to illustrate the proposed method, (3.1) has 
been chosen with an inhomogeneous term

$$
f(x, t)=2 x^{3}-15 x^{4} t^{4}
$$

for the set up equation (3.1). The solution of the equation (3.1) is to be obtained subject to the initial conditions

$$
u(x, 0)=u_{t}(x, 0)=0 .
$$

For the solution of this equation, we simply take the equation in operator form as given in (2.3) and using (2.4) to find the zeroth component of $u_{0}$ as

$$
u_{0}=x^{3} t^{2}-\frac{x^{4} t^{6}}{2}
$$

and obtain in succession $u_{1}, u_{2}, u_{3}, \ldots$ by using (2.8) to determine the other individual terms of the decomposition series, we find

$$
\begin{aligned}
u_{1} & =\frac{1}{2} L_{t}^{-1}\left(u_{0}^{2}\right)_{x x}+L_{t}^{-1} L_{x}\left(u_{0}\right)=\frac{x^{4} t^{6}}{2}-\frac{3 t^{8}}{14}-\frac{7 x^{5} t^{10}}{30}+\frac{x^{6} t^{10}}{104} \\
u_{2} & =\frac{1}{2} L_{t}^{-1}\left(\left(2 u_{1} u_{0}\right)_{x x}\right)+L_{t}^{-1} L_{x}\left(u_{1}\right) \\
& =\frac{3 t^{8}}{14}+\frac{7 x^{5} t^{10}}{30}-\frac{21 x^{6} t^{14}}{338}-\frac{7 x^{6} t^{12}}{66}+\frac{9 x^{7} t^{16}}{1183}+\cdots
\end{aligned}
$$

and so on. It is obvious that the self-canceling "noise" terms appear between various components, looking into the last term of $\mathfrak{u}_{0},(3.4)$, and first term of $u_{1},(3.5)$, are the self-canceling "noise" terms. We can readily observe that the second and third terms in $u_{1}$ and the first and second terms in $u_{2},(3.6)$, are self-canceling "noise" terms, and so on. Keeping the remaining noncanceled terms and using (2.5) leads immediately to the solution of (3.2) with initial conditions (3.3) given by $u(x, t)=t^{2} x^{3}$ which can be verified through substitution. It is worth noting that noise terms between components of series will be canceled, and the sum of these "noise" terms will vanish in the limit. This has been justified by $[4,17]$.

Problem 2. The equation we are now considering is

$$
u_{t t}=a\left[u^{3}+c u\right]_{x x}+u_{x x x x}
$$

which is sometimes called the cubic Boussinesq equation [13]. Without loss of generality, we can set $a=-2$ and $b=-1 / 2$. The problem (3.7) is given with the initial condition by

$$
u_{t t}=-2\left[u^{3}-\frac{1}{2} u\right]_{x x}+u_{x x x x}, \quad u(x, 0)=\frac{1}{x}, \quad u_{t}(x, 0)=-\frac{t}{x^{2}} \text {. }
$$


34 Explicit solutions of generalized nonlinear Boussinesq equations

Taking (3.8) in an operator form, the same as (2.3), we obtain

$$
u(x, t)=u(x, 0)+t u_{t}(x, 0)-2 L_{t}^{-1}(N(u))+L_{t}^{-1} L_{x x}(u),
$$

where $N(u)=\left[u^{3}-(1 / 2) u\right]_{x x}=\sum_{n=0}^{\infty} A_{n}$. For using the general formula of (2.7), one can generate $A_{n}$ Adomian polynomials as follow:

$$
\begin{aligned}
& A_{0}=\left[u_{0}^{3}-\frac{1}{2} u_{0}\right]_{x x}, \\
& A_{1}=\left[3 u_{0}^{2} u_{1}-\frac{1}{2} u_{1}\right]_{x x}, \\
& A_{2}=\left[3 u_{0} u_{1}^{2}+3 u_{0}^{2} u_{2}-\frac{1}{2} u_{2}\right]_{x x}, \\
& A_{3}=\left[u_{1}^{3}+6 u_{0} u_{1} u_{2}+3 u_{0}^{2} u_{3}-\frac{1}{2} u_{3}\right]_{x x},
\end{aligned}
$$

and so on for other polynomials.

The decomposition series solution $u(x, t)$ into $\sum_{n=0}^{\infty} u_{n}(x, t)$ in (2.4) and (2.8) with Adomian polynomials (3.10) yield the term-by-term components

$$
\begin{aligned}
u_{0} & =u(x, 0)+t u_{t}(x, 0)=\frac{1}{x}-\frac{t}{x^{2}} \\
u_{1} & =-2 L_{t}^{-1}\left(A_{0}\right)+L_{t}^{-1} L_{x}\left(u_{0}\right)=\frac{t^{2}}{x^{3}}-\frac{t^{3}}{x^{4}}-\frac{15 t^{4}}{x^{7}}+\frac{21 t^{5}}{5 x^{8}} \\
u_{2} & =-2 L_{t}^{-1}\left(A_{1}\right)+L_{t}^{-1} L_{x}\left(u_{1}\right) \\
& =\frac{t^{4}}{x^{5}}-\frac{t^{5}}{x^{6}}+\frac{15 t^{4}}{x^{7}}-\frac{273 t^{5}}{5 x^{8}}-\frac{6 t^{6}}{x^{7}}-\frac{272 t^{6}}{5 x^{9}}+\cdots
\end{aligned}
$$

and so on, in this manner the rest of the components of the decomposition series were obtained. Again, the self-canceling "noise" terms appear between various components for this problem, looking into the third term of $u_{1}$, (3.12), and third term of $u_{2},(3.13)$, are the self-canceling "noise" terms, and so on. Substituting (3.11), (3.12), and (3.13) into (2.5) gives the solution $u(x, t)$ in a series form and in a closed form by

$$
u(x, t)=\frac{1}{x+t} .
$$

This result can be verified through substitution.

Problem 3. The other equation we are considering is

$$
u_{t t}=[\ln u+c u]_{x x}+u_{x x x x}
$$


where $c$ is a constant. Without loss of generality, we can set $c=3$. The problem (3.15) is given with the initial conditions by

$$
u_{t t}=-2[\ln u+3 u]_{x x}+u_{x x x x}, \quad u(x, 0)=e^{x}, \quad u_{t}(x, 0)=2 e^{x} .
$$

Taking (3.16) as in (2.3), we get the form

$$
u(x, t)=u(x, 0)+t u_{t}(x, 0)+L_{t}^{-1}(N(u))+L_{t}^{-1} L_{x x}(u),
$$

where $N(u)=[\ln u+3 u]_{x x}=\sum_{n=0}^{\infty} A_{n}$ for this equation. For using the general formula of (2.7), one can generate $A_{n}$ Adomian polynomials as follow:

$$
\begin{aligned}
& A_{0}=\left[\ln u_{0}+3 u_{0}\right]_{x x}, \\
& A_{1}=\left[u_{1} u_{0}^{-1}+3 u_{1}\right]_{x x}, \\
& A_{2}=\left[u_{2} u_{0}^{-1}-\frac{u_{1}^{2}}{2} u_{0}^{-2}+3 u_{2}\right]_{x x}, \\
& A_{3}=\left[u_{3} u_{0}^{-1}-2 u_{1} u_{2} u_{0}^{-2}+\frac{u_{1}^{3}}{3} u_{0}^{-3}+3 u_{3}\right]_{x x},
\end{aligned}
$$

and so on for other polynomials.

The decomposition series solution $u(x, t)$ into $\sum_{n=0}^{\infty} u_{n}(x, t)$ in $(2.4)$ and (2.8) with Adomian polynomials (3.10) yield the term-by-term components

$$
\begin{aligned}
& u_{0}=e^{x}+2 t e^{x} \\
& u_{1}=L_{t}^{-1}\left(A_{0}\right)+L_{t}^{-1} L_{x}\left(u_{0}\right)=e^{x} \frac{(2 t)^{2}}{2 !}+e^{x} \frac{(2 t)^{3}}{3 !} \\
& u_{2}=L_{t}^{-1}\left(A_{1}\right)+L_{t}^{-1} L_{x}\left(u_{1}\right)=e^{x} \frac{(2 t)^{4}}{4 !}+e^{x} \frac{(2 t)^{5}}{5 !} \\
& u_{3}=L_{t}^{-1}\left(A_{2}\right)+L_{t}^{-1} L_{x}\left(u_{2}\right)=e^{x} \frac{(2 t)^{6}}{6 !}+e^{x} \frac{(2 t)^{7}}{7 !}
\end{aligned}
$$

and so on, in this manner the rest of the components of the decomposition series are obtained. Substituting (3.19) into (2.5) gives the solution $u(x, t)$ in a series form and in a closed form by

$$
u(x, t)=e^{x} e^{2 t}=e^{x+2 t}
$$

which can be easily verified.

\section{Conclusions}

In this paper, we calculated the explicit solution of certain generalized Boussinesq equation (1.1) by using the Adomian decomposition method. We 
demonstrated that the decomposition procedure is quite efficient to determine solutions in closed form when they exist. However, the method gives a simple and powerful tool for obtaining the solution without linearization nonlinear equations. It is also worth noting that the advantage of the decomposition methodology sometimes displays a fast convergence of the solution. It may be achieved by observing the self-canceling "noise" terms as discussed in $[4,17]$. In addition, the numerical results obtained by this method have illustrated a high degree of accuracy as discussed in $[12,16]$.

\section{Acknowledgement}

The author gratefully acknowledges the suggestions given by the referees.

\section{References}

[1] G. Adomian, A review of the decomposition method in applied mathematics, J. Math. Anal. Appl. 135 (1988), no. 2, 501-544. MR 89j:00046. Zbl 671.34053.

[2] Solving Frontier Problems of Physics: The Decomposition Method, Fundamental Theories of Physics, vol. 60, Kluwer Academic Publishers Group, Dordrecht, 1994. MR 95e:00026. Zbl 802.65122.

[3] Explicit solutions of nonlinear partial differential equations, Appl. Math. Comput. 88 (1997), no. 2-3, 117-126. CMP 1479 269. Zbl 904.35077.

[4] G. Adomian and R. Rach, Noise terms in decomposition solution series, Comput. Math. Appl. 24 (1992), no. 11, 61-64. CMP 1186 719. Zbl 777.35018.

[5] G. W. Bluman and J. D. Cole, The general similarity solution of the heat equation, J. Math. Mech. 18 (1969), 1025-1042. MR 45\#2334.

[6] J. Boussinesq, Théorie de L'ntumescene Liquide Appelée Onde Solitaire ou de Translation, se Propageant dans un Canal Rectangulaire, Comptes Rendus Acad. Sci. (Paris) 72 (1871), 755-759.

[7] Y. Cherruault, Convergence of Adomian's method, Kybernetes 18 (1989), no. 2, 31-38. MR 90i:65109. Zbl 697.65051.

[8] Y. Cherruault and G. Adomian, Decomposition methods: a new proof of convergence, Math. Comput. Modelling 18 (1993), no. 12, 103-106 (1994). MR 94k:65083. Zbl 805.65057.

[9] P. A. Clarkson, New exact solutions of the Boussinesq equation, European J. Appl. Math. 1 (1990), no. 3, 279-300. MR 92d:35244. Zbl 721.35074.

[10] P. A. Clarkson and M. D. Kruskal, New similarity reductions of the Boussinesq equation, J. Math. Phys. 30 (1989), no. 10, 2201-2213. MR 90m:35161. Zbl 698.35137.

[11] P. Deift, C. Tomei, and E. Trubowitz, Inverse scattering and the Boussinesq equation, Comm. Pure Appl. Math. 35 (1982), no. 5, 567-628. MR 84b:35105. Zbl 489.35073.

[12] D. Kaya, An application of the decomposition method for second order wave equations, Int. J. Comput. Math. 75 (2000), no. 2, 235-245. MR 2001d:65134. Zbl 0964.65113.

[13] T. J. Priestley and P. A. Clarkson, Symmetries of a generalized Boussinesq equation, Tech. report, IMS Technical Report UKC/IMS/59, 1996. 
[14] A. Répaci, Nonlinear dynamical systems: on the accuracy of Adomian's decomposition method, Appl. Math. Lett. 3 (1990), no. 4, 35-39. MR 91m:34021. Zbl 719.93041.

[15] P. Rosenau, Dynamics of dense lattices, Phys. Rev. B (3) 36 (1987), no. 11, 5868-5876. MR 88m:82046.

[16] A. M. Wazwaz, The decomposition method for approximate solution of the Goursat problem, Appl. Math. Comput. 69 (1995), no. 2-3, 299-311. CMP 1326 677. Zbl 826.65077.

[17] Necessary conditions for the appearance of noise terms in decomposition solution series, Appl. Math. Comput. 81 (1997), no. 2-3, 265-274. Zbl 882.65132.

[18]_A new algorithm for calculating Adomian polynomials for nonlinear operators, Appl. Math. Comput. 111 (2000), no. 1, 53-69. CMP 1745908.

[19] V. E. Zakharov and A. B. Shabat, A scheme for integrating the nonlinear equations of mathematical physics by the method of the inverse scattering problem. I, Funct. Anal. Appl. 8 (1974), no. 3, 43-53.

Doğan Kaya: Department of Mathematics, Firat University, Elazig, 23119, Turkey E-mail address: dkaya@firat.edu.tr 


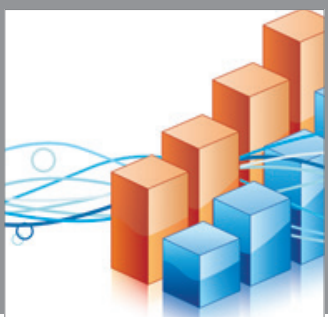

Advances in

Operations Research

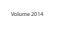

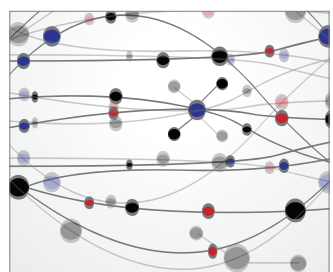

\section{The Scientific} World Journal
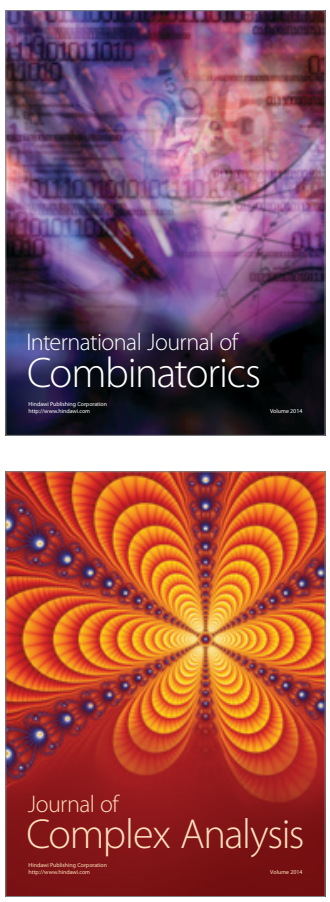

International Journal of

Mathematics and

Mathematical

Sciences
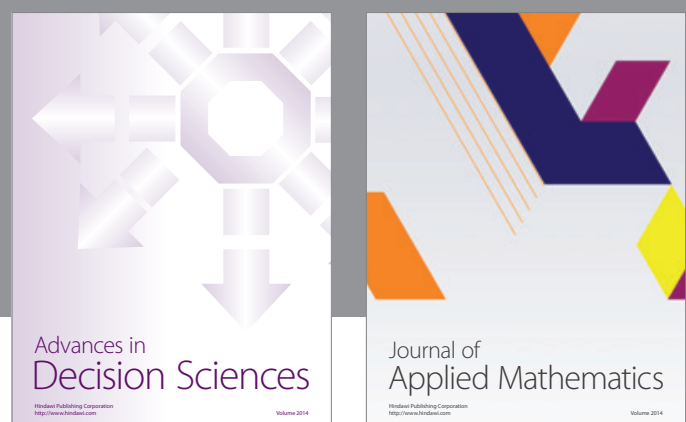

Journal of

Applied Mathematics
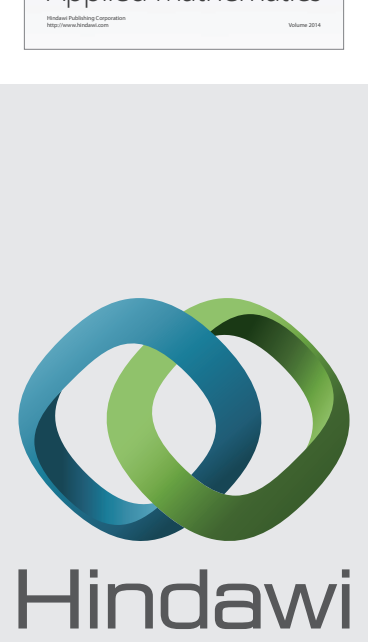

Submit your manuscripts at http://www.hindawi.com
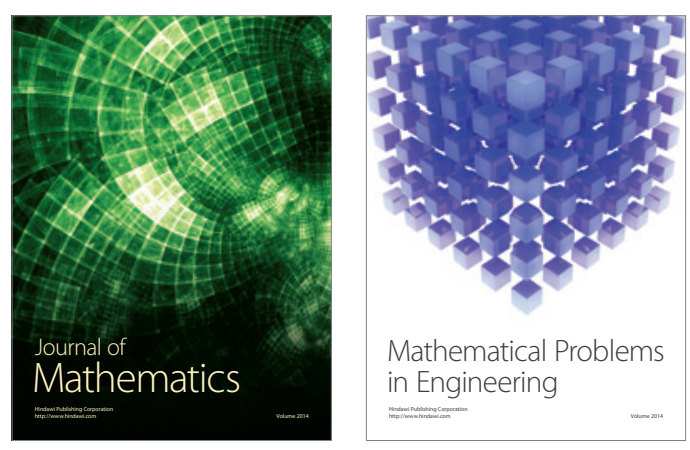

Mathematical Problems in Engineering
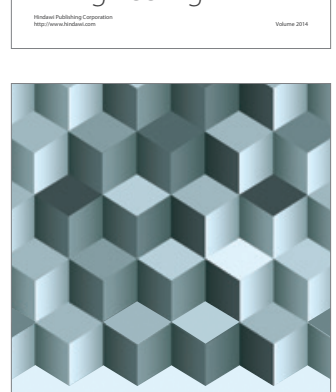

Journal of

Function Spaces
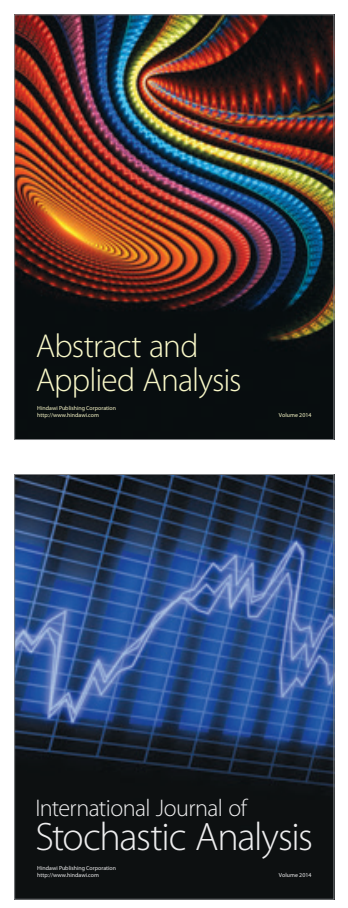

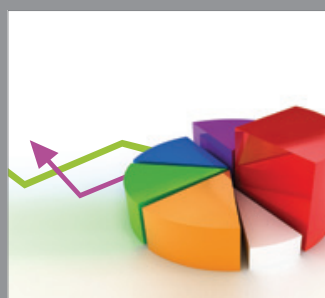

ournal of

Probability and Statistics

Promensencen
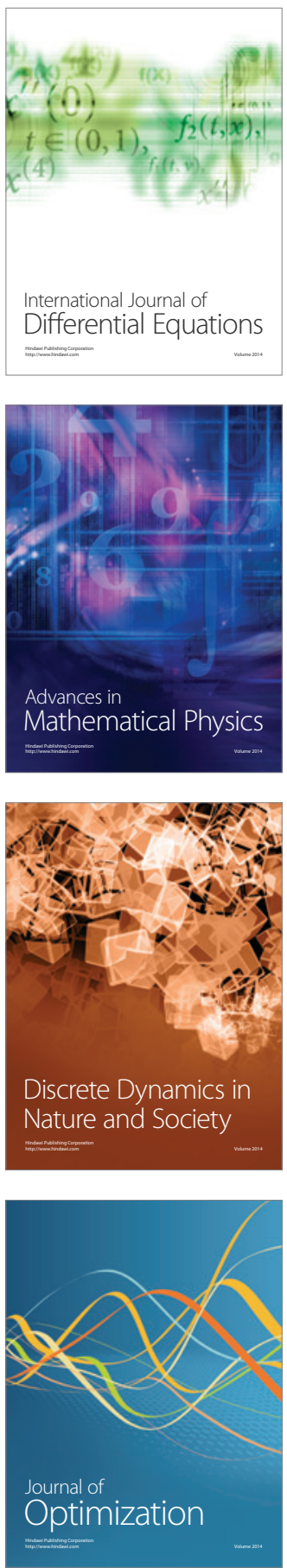